\title{
Apego y estrés en niños con Diabetes tipo 1 y sus madres
}

\section{Attachment and stress in children with type 1 Diabetes and their mothers}

\author{
Costa-Cordella, Stefanella ${ }^{\mathrm{a}, \mathrm{f}}$, Luyten, Patrick ${ }^{\mathrm{a}, \mathrm{b}, \mathrm{f}}$, Giraudo, Franco $^{\mathrm{c}, \mathrm{d}}$, \\ Mena, Francisca ${ }^{\mathrm{d}, \mathrm{f}}$, Shmueli-Goetz, Yael ${ }^{\mathrm{a}, \mathrm{e}, \mathrm{f}}$, Fonagy, Peter ${ }^{\mathrm{a}, \mathrm{e}, \mathrm{f}}$
}

\author{
aResearch Department of Clinical, Educational and Health Psychology, University College London, Londres, Reino Unido \\ bUniversity of Leuven, Leuven, Bélgica \\ 'Facultad de Medicina, Universidad de Chile, Santiago, Chile \\ 'Fundación Diabetes Juvenil, Santiago, Chile \\ eThe Anna Freud Centre, Londres, Reino Unido \\ fPsicólogo (a)
}

Recibido: 17 de abril de 2019; Aceptado: 24 de octubre de 2019

¿Qué se sabe del tema que trata este estudio?

La diabetes mellitus tipo 1 (DM1) es causa de estrés, para paciente y cuidador. Es común en cuidadores el estrés parental y postraumático y éste afecta la condición del/la niño/a: mayores niveles de psicopatología y baja adherencia al tratamiento. Un sistema bio-comportamental de regulación estrés, clave en relaciones de cuidado, es el Apego.
¿Qué aporta este estudio a lo ya conocido?

Las estrategias de apego tanto maternas como infantiles son significativas en los resultados de DM1 (RD) aunque con diferencias de género inesperadas. El apego evitativo materno mostró asociación con RD desfavorable y el apego infantil seguro relación con RD favorables.

\section{Resumen}

Objetivo: Comprender la relación entre apego y diabetes y el rol mediador del estrés en niños con diabetes mellitus tipo 1 (DM1) y sus madres. Material y Método: Se aplicaron Instrumentos de evaluación correspondientes a Escalas de Apego (ECR-R), Estrés percibido (PSS), Seguridad (SS) y Estrés en niños ( $\mathrm{SiC}$ ), como medidas de autoreporte completadas por niños(as) y sus madres. Se analizaron variables demográficas, tiempo de inicio de diabetes, y el promedio de las ultimas 3 mediciones de Hemoglobina glicosilada HbA1c como parámetro del control metabólico del último año. Resultados: Las estrategias de apego maternas e infantiles y el estrés materno mostraron una asociación significativa con los resultados de la diabetes del niño(a), aunque con importantes diferencias de género. Conclusiones: Las estrategias de apego, infantiles y maternas, son relevantes en el curso de la diabetes.
Palabras clave: diabetes tipo 1; apego; estrés, vínculos emocionales; madre; cuidadores; niños; niñas 


\begin{abstract}
Objective: To understand the relationship between attachment and diabetes and the role of stress mediators in children with type 1 diabetes (T1D) and their mothers. Material and Method: The following assessment instruments were applied as self-report measures: Attachment Scale (ECRR), Perceived Stress Scale (PSS), Security Scale (SS), and the Stress in Children (SiC) questionnaire, which were completed by children and their mothers. We analyzed demographic variables, diabetes onset time, and the average of the last three glycosylated hemoglobin (HbAlc) measurements as a parameter of metabolic control in the last year. Results: Attachment strategies of both mother and child, as well as maternal stress, showed a significant association with the child's diabetes outcomes, although with important gender differences. Conclusions: Both mother and child attachment strategies are relevant aspects of the T1D course.
\end{abstract}

\section{Keywords:}

Type 1 diabetes; attachment; stress, emotional bonds; mother; caregiver; children

\section{Introducción}

Existe creciente evidencia acerca del rol del estrés en el curso de la diabetes mellitus tipo 1 (DM1)1. La relación entre estrés y DM1 se entiende como bidireccional (figura 1): la carga por el diagnóstico y el tratamiento de la DM1 son causa de estrés ${ }^{2}$ y a su vez el estrés puede ser causa de desregulaciones en el control de la diabetes de manera directa (vía mecanismos fisiológicos ligados al control metabólico $)^{2} \mathrm{o}$ indirecta (mediante una disminución en la adherencia al tratamiento) $)^{3}$.

En la infancia la relación entre estrés y DM1 involucra también a los cuidadores, quienes poseen la mayor responsabilidad por el tratamiento. La evidencia muestra que padres y madres de niños(as) con T1D sufren de estrés parental y estrés postraumático ${ }^{4}$. A su vez, el estrés parental afecta el curso de la condición en el niño(a), asociándose a mayores niveles de psicopatología y baja adherencia al tratamiento ${ }^{5}$.

Un sistema bio-comportamental para la regulación del estrés es el apego ${ }^{6}$. La teoría del apego ${ }^{7}$ plantea la necesidad humana innata de generar lazos afectivos cercanos, que se manifiesta especialmente en momentos de estrés. El sistema de apego (SA) se activa frente a una amenaza que puede ser externa (como un desastre natural) o interna (como una enfermedad)8. En la infancia, el estrés vivenciado por el niño(a) activa el SA hacia su cuidador. En el cuidador, la percepción de amenaza hacia el niño(a) activa su sistema de cuidado, que implica ayudarlo a regular su respuesta de estrés ${ }^{8}$.

A partir de la repetición de interacciones de regulación interpersonal del estrés se genera un sistema de expectativas acerca del sí mismo y los otros o "modelo interno"12 del funcionamiento de las relaciones de cuidado, dando origen a un patrón o estrategia de apego.

Se han identificado 4 estrategias de apego predominantes: seguro, inseguro evitativo, inseguro ansioso y desorganizado?.

Los niños(as) con apego seguro expresan su estrés sabiendo que será reconocido y calmado por el cuida- dor. En presencia de éste el estrés disminuye, desactivándose el SA.

En el patrón evitativo, los niños tienden a no mostrar signos de estrés aun cuando fisiológicamente esté activado $^{10}$. Presentan una sobre-regulación afectiva: señales de estrés son suprimidas para mantenerse cerca del cuidador, temiendo su rechazo frente a la expresión de emociones negativas. No buscan al cuidador ante situaciones de estrés ${ }^{15}$.

Contrariamente, el patrón ansioso se caracteriza por una exageración de la expresión de molestia para lograr la atención del cuidador, quien suele responder

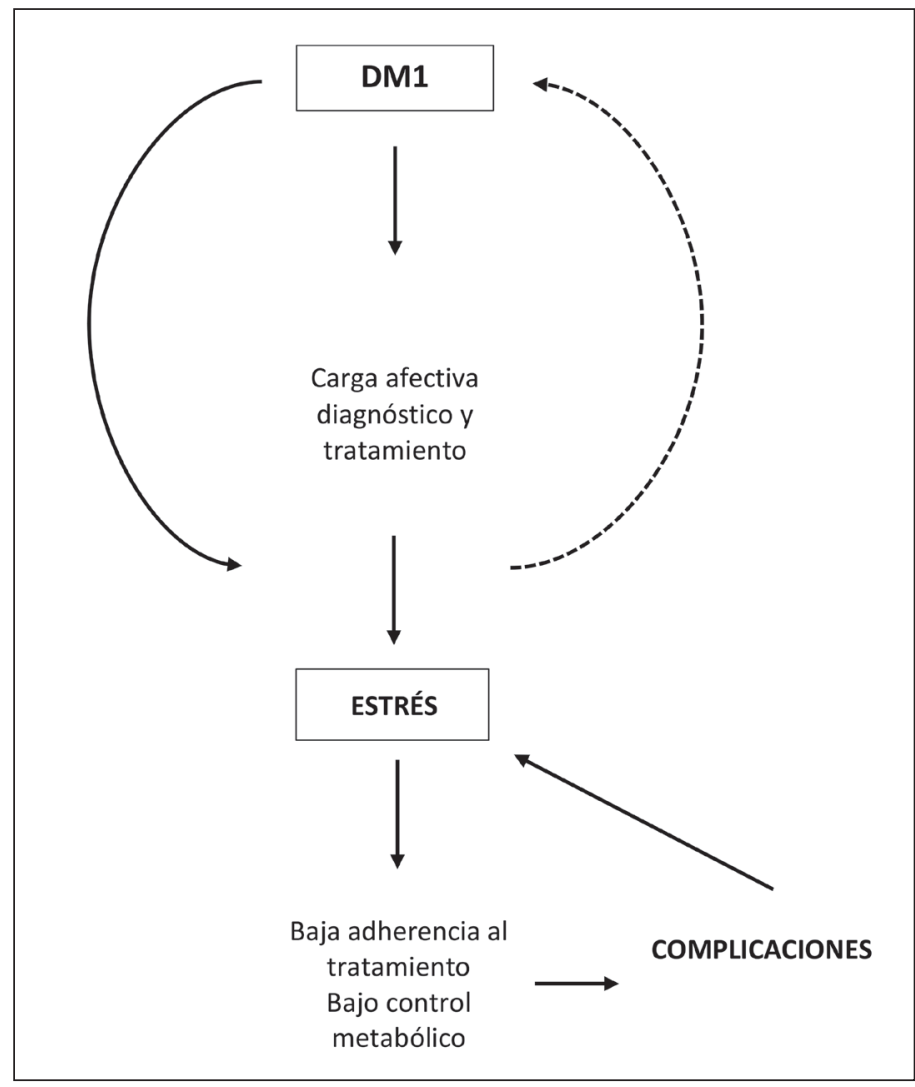

Figura 1. Relación bidireccional entre Diabetes (DM1) y Estrés. 
de manera inconsistente. Las emociones están subreguladas y el estrés se manifiesta con intensidad. Sin embargo, la cercanía con el cuidador no provee calma y la respuesta al estrés (y por ende, el SA) se mantienen $\operatorname{activados}^{15}$.

Niños(as) con apego desorganizado muestran un patrón inconsistente producto de repetidas experiencias con cuidadores amenzantes e incapaces de reconocer y calmar el estrés en sus niños(as), resultando en una activación crónica de los estados emocionales negativos $^{15}$.

Según estudios longitudinales1 1 el apego es importante y puede ser medido durante todo el ciclo vital, con un $69-75 \%$ de concordancia entre la infancia y adultez. Las mediciones de apego pueden ser categóricas y clasificar al sujeto en una estrategia de apego específica o dimensionales, y situar al sujeto en un punto dentro de un continuo entre seguridad e inseguridad en el apego ${ }^{12}$.

Las diferencias individuales en el apego han mostrado ser relevantes en el curso de la DM1 ${ }^{13}$. Se ha visto que pacientes con apego evitativo presentan menor adherencia al tratamiento que pacientes con apego seguro y ansioso ${ }^{20}$. Los padres de niños(as) con DM1 con apego evitativo presentan mayor estrés parental e impacto más negativo de la DM1 que cuidadores con otros patrones de apego ${ }^{14}$.

La relación médico-paciente media la asociación entre apego y adherencia al tratamiento. Un estudio poblacional en adultos con DM1 y DM2 $(\mathrm{N}=4,095)^{20}$ evidenció que pacientes con apego seguro presentaron mayores niveles de colaboración con el equipo médico que pacientes con otros estilos de apego, demostrando una mejor adherencia al tratamiento.

El SA tiene un efecto directo en la respuesta al estrés ${ }^{15}$ y el estrés se regula en un proceso cooperativo con las figuras de apego ${ }^{23}$. Por lo tanto, la relación entre apego y estrés es bidireccional, existiendo dos posibles caminos: el estrés activa el SA, éste regula la respuesta al estrés y se retoma la homeostásis o, por el contrario, su activación inefectiva desencadena la activación de las llamadas "estrategias de apego secundario" (hipo e hiperactivación $)^{16}$ que son insuficientes para regular el estrés (figura 2). Esto desencadena una activación crónica del estrés que a su vez reactiva las estrategias de apego secundario, en un círculo vicioso que generaría

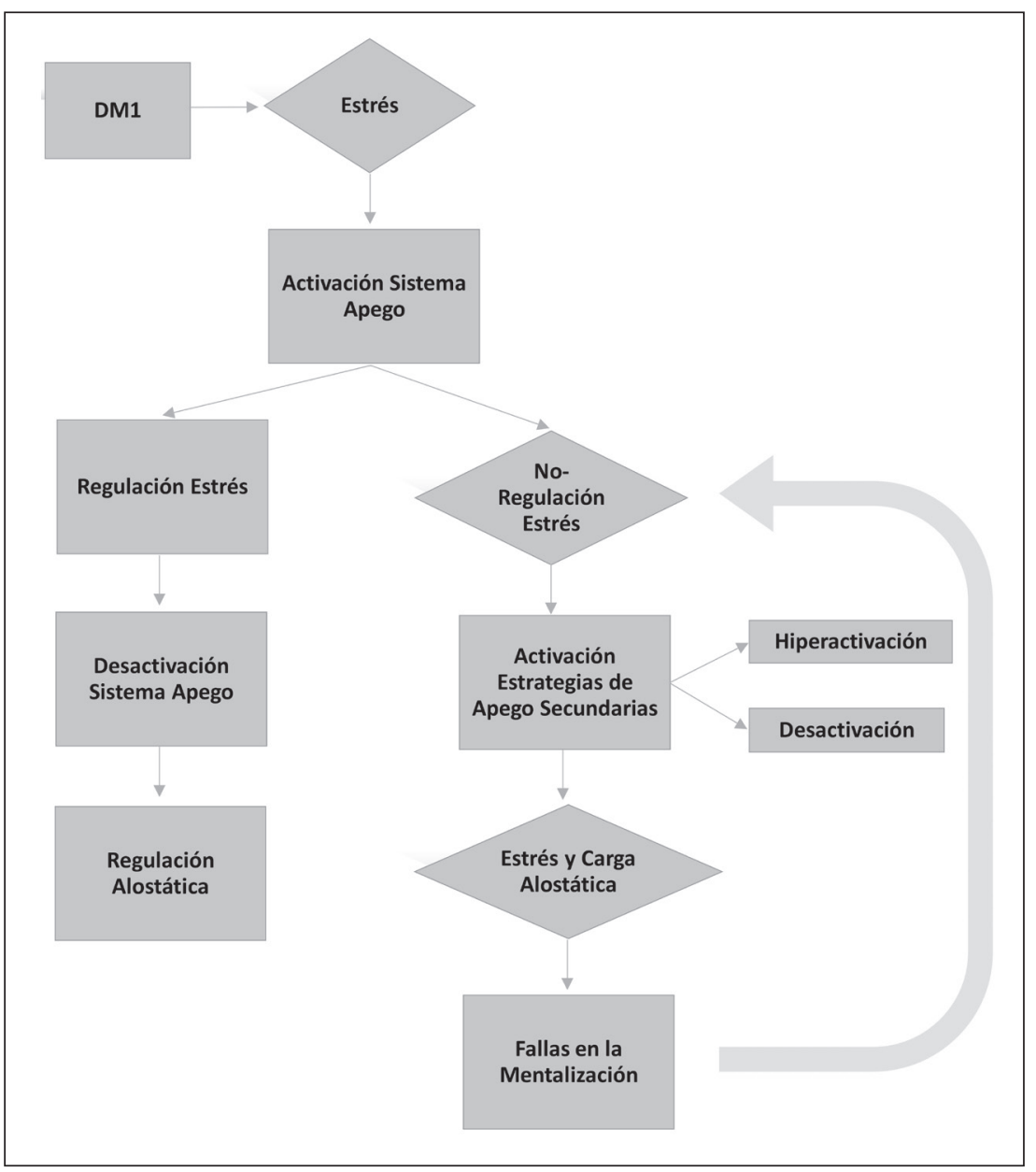

Figura 2. Proceso activación/desactivación apego y estrés. 
una sobrecarga alostática ${ }^{17}$, la cual explicaría el origen de ciertas enfermedades relacionadas con la desregulación del principal sistema endocrinológico para la regulación del estrés, el eje Hipotalámico Pituitario Adrenal (HPA) ${ }^{18}$.

En DM1, la activación crónica del SA generada por el estrés puede ser fuente de desregulaciones metabólicas que resulten en un control desfavorable.

Se sabe que un período particularmente sensible a la desregulación es la adolescencia ${ }^{19}$. Por este motivo, la población de adolescentes con DM1 ha sido ampliamente estudiada, a diferencia de la etapa anterior. Éste es un importante vacío ya que en la pre-adolescencia se sientan las bases del autocuidado ${ }^{20}$. Además, se sabe que una causa de la no-adherencia durante la adolescencia es la transferencia poco gradual de las responsabilidades desde los padres hacia el adolescente ${ }^{21}$. Se hace relevante por tanto investigar a la población de niños/as pre-adolescentes.

Además, no existen estudios que consideren cómo los patrones de apego de niños con DM1 y de sus cuidadores influyen en los resultados de la diabetes (RD). También, el rol del estrés como mediador en la relación entre apego y diabetes no ha sido estudiado.

En respuesta, se investigaron las siguientes hipótesis:

1) Mayor seguridad en el apego, tanto en cuidadores como en niños, se asocia a RD favorables.
2) Mayores niveles de estrés, tanto en niños(as) como en cuidadores, se asocia a RD desfavorables.

3) El estrés (del cuidador/a y/o del niño/a) media la relación entre apego (cuidador/a y/o niño/a) y RD.

Este estudio tuvo como objetivo comprender la relación entre apego y diabetes y el rol mediador del estrés en niños con DM1 y sus madres*. Mediante un diseño transversal, se utilizaron datos empíricos provenientes de medidas de autoreporte completadas por niños(as) entre 8 y 12 años y sus madres.

\section{Material y Método}

Se realizó un estudio observacional. Niños(as) y sus madres, socios de la Fundación Diabetes Juvenil de Chile (FDJ), fueron reclutados vía correo electrónico. A aquellos que demostraron interés se les envío hoja de información, consentimiento informado y enlace hacia el sitio web del estudio (plataforma RedCap).

Este estudio fue aprobado por el comité de ética de University College London (Reino Unido; UCL Research Ethics Committee) número 8899/001.

* Sólo con el fin de resguardar la homogeneidad en la muestra se incluyó exclusivamente a madres como cuidadores principales, considerando los datos de la encuesta CASEN (2011), que señala que en Chile sólo un $4 \%$ de los cuidadores principales son padres.

\section{Tabla 1. Instrumentos Utilizados y Variables}

\begin{tabular}{ll} 
Variable & Instrumento \\
\hline Diabetes (niño/a) & Hemoglobina Glicosilada (HbA1c)
\end{tabular}

Descripción

Indica concentración promedio de glucosa en sangre durante los últimos 90-120 días. Es considerado un indicador fundamental de control glicémico ${ }^{30}$. Se recomienda en pacientes pedriátricos valores inferiores a 7\%; niveles mayores se asocian a complicaciones crónicas $^{30}$. Para el análisis se utilizó el promedio de las tres últimas mediciones de $\mathrm{HbA} 1 \mathrm{c}$, como parámetro del control metabólico del último año

Inventario de calidad de vida pediátrica ${ }^{31}$ (Módulo diabetes PEDSQoL 3.2)

\section{Apego (Madre)}

Escala de Experiencia en Relaciones Cercanas-revisada ${ }^{33}$ (ECR-R) versión chilena ${ }^{34}$

Estrés (madre) Escala de estrés percibido ${ }^{35}$ (PSS) versión chilena ${ }^{36}$ :

Apego (niño/a) Escala de Seguridad ${ }^{37}$ (SS) versión latinoamericana ${ }^{38}$

Stress (niño/a) Estrés en Niños $(\mathrm{SiC})^{39}$ versión chilena ${ }^{40}$
Mide calidad de vida asociada específicamente a DM1 informada por los padres, indicador psicosocial fundamental sugerido en guías clínicas internacionales ${ }^{32}$. Evalúa la integración de la condición en la vida del niño/a. Las respuestas están en escala Likert de 5 puntos donde mayores puntuaciones significan mejor calidad de vida. En este estudio se utilizó como indicador de RD

Evalúa estilos de apego adulto a través de ítems calificados con formato Likert de 7 puntos. Evalúa dos dimensiones del apego: ansiedad y evitación (18 ítems cada escala)

Cuestionario de 14 ítems. Se pide informar con qué frecuencia se sintió de una manera particular durante el mes pasado. Puntuaciones más altas representan mayores niveles de estrés percibido, de $1=$ nunca a $4=$ muy a menudo

Cuestionario de 15 elementos que evalúa la percepción del niño(a) sobre la seguridad del apego en relación con su madre. Los ítems se puntúan de 1 a 4 . Mayores puntuaciones indican mayor percepción de seguridad

Cuestionario de 21 ítems para niños en edad escolar. Evalúa angustia percibida, bienestar, afrontamiento y apoyo social. Mide frecuencia con que sujetos pensaron o sintieron de cierta manera durante el último mes, desde "nunca" hasta "muy a menudo". Puntuaciones más altas significan niveles más altos de estrés 


\section{Criterios de inclusión}

Niños y niñas (8-12 años) junto a su madre, mínimo un año desde diagnóstico de DM1.

\section{Criterios de exclusión}

Diagnóstico psiquiátrico (niño/a o madre), concomitancia otras enfermedades crónicas.

Los participantes fueron evaluados con los siguientes instrumentos (tabla 1).

\section{Análisis de datos}

Se realizaron correlaciones de Pearson entre las variables de estudio utilizando SPSS 24. Cada correlación

Tabla 2. Descripción sociodemográfica

\begin{tabular}{lcc}
\hline & Media (SD) ○ \% & $n$ \\
\hline Información madre & & \\
Edad (Años) & $38,9( \pm 7,07)$ & 77 \\
Nivel educacional (\%) & & \\
Educación básica completa & $2,6 \%$ & 2 \\
Educación media completa & $14,3 \%$ & 11 \\
Educación técnica incompleta & $9,1 \%$ & 7 \\
Educación técnica completa & $23,4 \%$ & 18 \\
Educación universitaria incompleta & $14,3 \%$ & 11 \\
Educación universitaria completa & $27,3 \%$ & 21 \\
Estudios de postgrado & $9,1 \%$ & 7 \\
Nivel socioeconómico (\%) & & \\
Alto & $44,2 \%$ & 34 \\
Medio & $48,1 \%$ & 37 \\
Medio-Bajo & $6,5 \%$ & 5 \\
Bajo & $1,3 \%$ & 1 \\
Información niños/as & & \\
Edad (Años) & $10,12( \pm 1,57)$ & 77 \\
Género (\%) & & \\
Femenino & $55,8 \%$ & 43 \\
Masculino & $44,2 \%$ & 34 \\
Tiempo de inicio diabetes (meses) & $38,7( \pm 31,8)$ & 77 \\
Hba1c & $8( \pm 1,2)$ & 77 \\
\hline
\end{tabular}

fue analizada en niños y niñas de manera independiente, considerando, de manera exploratoria, la posibilidad de que apareciesen diferencias de género en alguna de las relaciones. Lo anterior se decidió en base a los resultados de estudios previos ${ }^{22}$ en diabetes y psicología. ${ }^{* *}$

Se utilizó Hayes (2012) macros PROCESS para SPSS 24 para explorar el rol mediador del estrés (del niño(a) y del cuidador) en la relación entre apego y diabetes.

\section{Resultados}

\section{Descripción muestra}

Se reclutó un total de 77 duplas madres-hijo(a), distribuidos en $44,2 \%$ niños y $55,8 \%$ niñas. Las edades promedio de los niños considerando ambos sexos fue $10,12 \pm 1,57$ años y la de sus cuidadores $38,95 \pm 7,07$ años. Se les preguntó además por nivel educacional y nivel socioeconómico (tabla 2). Ninguna de las variables de estudio mostró correlación significativa con las variables sociodemográficas.

\section{Correlaciones}

A continuación se describen los resultados de los análisis de correlaciones de Pearson mostrados en la tabla 3.

\section{Apego cuidador y diabetes}

En los niños se encontró una alta correlación negativa entre niveles de apego ansioso y evitativo del cuidador y calidad de vida específica de diabetes en los niños (D-QoL) $(r=-0,50, p<0,01 ; r=-0,42, p<0,01$, respectivamente). Es decir, en los niños, a mayor ansiedad o evitación de la madre menor D-QoL.

** En este estudio las diferencias de género no fueron abordadas desde el diseño y formulación teórica del mismo, por lo que no son parte de las hipótesis de trabajo.

Tabla 3. Correlación entre Apego del niño/a (SS), Apego de la Madre (ECR) y Resultados de la Diabetes (Calidad de vida pediátrica [PEDSQoL] y hemoglobina glicosilada [HbA1c])

\begin{tabular}{|c|c|c|c|c|c|c|c|c|c|c|}
\hline & & \multicolumn{3}{|c|}{$\begin{array}{c}\text { Todos } \\
\text { Escala ECR (Apego madre) }\end{array}$} & \multicolumn{3}{|c|}{$\begin{array}{c}\text { Niños } \\
\text { Escala ECR (Apego madre) }\end{array}$} & \multicolumn{3}{|c|}{$\begin{array}{c}\text { Niñas } \\
\text { Escala ECR (Apego madre) }\end{array}$} \\
\hline & & Evitación & Ansiedad & $\begin{array}{l}\text { SS (Apego } \\
\text { niño/a) }\end{array}$ & Evitación & Ansiedad & $\begin{array}{l}\text { SS (Apego } \\
\text { niño/a) }\end{array}$ & Evitación & Ansiedad & $\begin{array}{c}\text { SS (Apego } \\
\text { niño/a) }\end{array}$ \\
\hline \multirow{2}{*}{$\begin{array}{l}\text { Dimensiones } \\
\text { PEDSQoL }\end{array}$} & PEDSQoL Total & $-0,502 * *$ & $-0,420 * *$ & $0,203^{*}$ & $-0,322 *$ & $-0,285^{*}$ & 0,194 & $-0,261$ & $-0,548 * *$ & 0,166 \\
\hline & $\mathrm{HbA} 1 \mathrm{c}$ & $-0,003$ & $-0,022$ & $-0,166$ & $0,291^{\ddagger}$ & $-0,017$ & $-0,358 *$ & $-0,334^{*}$ & $-0,056$ & 0,096 \\
\hline
\end{tabular}

Nota: ${ }^{\ddagger} p<0,1 ;{ }^{*} p<0,05,{ }^{* *} p<0,01$ 
En las niñas, en cambio, no aparecen correlaciones significativas entre apego del cuidador y D-QoL pero la variable $\mathrm{HbA} 1 \mathrm{c}$ mostró una correlacion negativa con evitación del cuidador $(\mathrm{r}=-0,33, \mathrm{p}<0,01)$. Es decir, a mayor evitación, mejor control metabólico en las niñas.

\section{Apego infantil y diabetes}

D-QoL no mostró correlación significativa con Apego infantil.

HbA1c mostró una correlación negativa con seguridad en el apego infantil en niños $(r=-0,35, p<0,05)$ pero no en niñas. Es decir, a mayor seguridad en el apego infantil, mejores RD en niños.

\section{Stress del cuidador y diabetes}

El estrés percibido del cuidador (PSS) mostró una correlación negativa con D-QoL $(r=-0,32, p<0,05)$ en niños, pero no en niñas.

En niñas, PSS presentó una correlación negativa con HbA1c $(r=-0,41, p<0,05)$, implicando que a mayor estrés materno, mejor RD en niñas, contrario a lo esperado.

\section{Stress del niño y diabetes}

Sopresivamente, ninguna de las escalas de estrés en niños mostró correlación con HbA1c o D-QoL.

\section{Modelos mediacionales \\ Ningún modelo mediacional resultó significativo. Discusión}

Se investigó la relación entre apego, estrés y diabetes en niños(as) con DM1 y sus madres. Los resultados indican que las estrategias de apego maternas e infantiles son significativas en los RD aunque con importantes diferencias de género que no estaban previstas. En niños, acorde con lo esperado, el apego evitativo materno mostró asociación con $\mathrm{RD}^{* * *}$ desfavorables mientras que el apego infantil seguro se relacionó con RD favorables. Esto es concordante con estudios anteriores donde pacientes con apego inseguro mostraron $\mathrm{RD}$ desfavorables ${ }^{20}$; especialmente aquellos con apego inseguro-evitativo ${ }^{19,20}$. Particularmente, en este estudio se analizó el apego de la madre. Se sabe que madres con apego inseguro-evitativo tienden a rechazar las expresiones de vulnerabilidad de sus hijos, poniendo énfasis en la auto-eficacia y el logro ${ }^{23}$. Así, estos niños aprenden a omitir la expresión de necesidades de apego por miedo al rechazo materno, logrando autonomía tempranamente a un alto costo a nivel socioemocional (se suprime la expresión de emociones y se tiende al

\footnotetext{
*** Se hablará de RD para referirse a los indicadores HbAlc y PEDSQoL indistintamente, ambos como variables proxy de "Resultados de Diabetes".
}

aislamiento social) ${ }^{10}$ y a nivel fisiológico (respuesta de estrés se activa sin manifiestarse en la conducta, por lo que no se reconoce haciéndose más difícil de regular) ${ }^{16}$. Nuestros resultados indican que este sistema interaccional podría afectar de manera diferente según género y esto nos obliga a discutir los resultados considerando esta variable a pesar de no haberla contemplado en la formulación teórica del problema de investigación.

En los niños, la desregulación del estrés resultante de la estrategia evitativa materna se relaciona con RD desfavorables. Por el contrario, en las niñas la desatención materna empujaría hacia una autonomía temprana suficiente para controlar la diabetes (al menos en el corto plazo), aunque probablemente esta estrategia se caracterice por una autosuficiencia compulsiva que, como tal, tienda a fallar en el largo plazo.

Esta diferencia de género podría comprenderse desde una perspectiva antropológica y social. En primer lugar, el cuidado del propio cuerpo es experienciado de distinta manera en mujeres que en hombres. En las culturas latinoaméricanas las mujeres son percibidas como cuidadoras innatas (mujer y madre como sinónimo $)^{24}$ de otros y de ellas mismas. En segundo lugar, la cultura occidental valora el control del propio cuerpo especialmente en mujeres ${ }^{25}$, lo que se refleja en el valor que adquieren la delgadez y el control de los impulsos sexuales al ligarse a lo femenino ${ }^{38}$. Y por último, el tratamiento de DM1 promueve y premia el control, por su evidente valor en el equilibrio metabólico. Estos tres puntos dan cuenta de la gratificación que podría significar para las niñas el cumplimiento del mandato cultural de control que se entrelaza con la evitación materna y su imperativo de no mostrarse vulnerable.

Aquella evitación materna que en niños parece resultar en un descuido de la diabetes, en niñas, por el contario, parece fortalecer la idea de cuidadoras de sí mismas. Sin embargo, es probable que esta estrategia eficiente para el logro de RD positivos (en el corto plazo), sea propensa a fallar en el largo plazo, por su alto costo psicosocial y metabólico (desregulación). Por ejemplo, se ha visto que niñas que presentaban buen control en la infancia (corto plazo) desarrollan trastornos de conducta alimentaria (TCA) en la adolescencia (largo plazo ${ }^{26}$. El control riguroso de la comida implicado en el tratamiento de diabetes ha sido identificado como el principal factor de riesgo para TCA en población con $\mathrm{DM}^{40}$. Además, mujeres presentan el doble de riesgo que hombres de desarrollar TCA27. Nuestros resultados apoyan la noción de que una estrategia eficaz para el control de la diabetes en el corto plazo, puede compartir la raíz con trastornos mentales en el largo plazo, en un contexto de apego inseguro.

Lo anterior, además, es concordante con nuestros resultados respecto del estrés. Mayor estrés materno 
se asoció a RD desfavorables en niños, mientras que en niñas se encontró el patrón opuesto: a mayor estrés materno, RD más favorables. Esto demuestra el rol fundamental del estrés materno en el desarrollo del autocuidado infantil. Estrés infantil, en cambio, no mostró asociaciones significativas con apego ni diabetes.

La discusión sobre las diferencias de género debe ser abordada con precaución. Es posible que la variable sexo juegue un rol importante, sobre todo considerando las diferencias en los desarrollos puberales femeninos y masculinos; sin embargo, nuestro diseño no nos permite ahondar en este punto. Futuros estudios debiesen considerar incluir una evaluación del estadio de pubertad de los participantes utilizando la Escala de Tanner ${ }^{28}$.

Con respecto a la tercera hipótesis -el rol mediador del estrés- no se encontraron resultados significativos. Esto podría explicarse en parte por las limitaciones de este estudio, las que se discuten a continuación.

El diseño observacional, transversal y con medidas de auto-reporte no permiten extraer relaciones causales de nuestros resultados. Futuras investigaciones debieran considerar diseños longitudinales para así desentrañar la eficacia de las estrategias a largo plazo. Además, futuros estudios se beneficiarían usando procedimientos experimentales y medidas biológicas de estrés. Otra limitación importante de nuestro estudio es la falta de distinción en las técnicas de medición de HbA1c que podría afectar los resultados. Si bien es probable que la mayoría de los participantes haya utilizado el método DCA 2000+ para HbA1c capilar (ya que es el más utilizado en $\mathrm{Chile}^{29}$ ) no podemos afirmarlo con certeza. Futuros estudios debiesen establecer esta distinción de manera clara y considerarla en los análisis.

Por último, nuestros resultados reflejan que las distintas formas de vincularse interpersonalmente en relaciones de cuidado son importantes en salud; tanto en considerar a la madre (y su propia subjetividad) en los tratamientos pediátricos, como en la relevancia de la relación médico-paciente.

\section{Responsabilidades Éticas}

Protección de personas y animales: Los autores declaran que los procedimientos seguidos se conformaron a las normas éticas del comité de experimentación humana responsable y de acuerdo con la Asociación Médica Mundial y la Declaración de Helsinki.

Confidencialidad de los datos: Los autores declaran que han seguido los protocolos de su centro de trabajo sobre la publicación de datos de pacientes.

Derecho a la privacidad y consentimiento informado: Los autores han obtenido el consentimiento informado de los pacientes y/o sujetos referidos en el artículo. Este documento obra en poder del autor de correspondencia.

\section{Conflicto de intereses}

Los autores declaran no tener conflicto de intereses.

\section{Referencias}

1. Surwit RS, Schneider MS. Role of stress in the etiology and treatment of diabetes mellitus. Psychosom Med. 1993;55(4):380-93. https://doi. org/10.1097/00006842-199307000-00005.

2. Butwicka A, Frisén L, Almqvist C, Zethelius B, Lichtenstein P. Risks of psychiatric disorders and suicide attempts in children and adolescents with type 1 diabetes: a population-based cohort study. Diabetes Care 2015;38(3):453-9. https:// doi.org/10.2337/dc14-0262.

3. Martinez K, Frazer SF, Dempster M, Hamill A, Fleming H, McCorry NK. Psychological factors associated with diabetes self-management among adolescents with Type 1 diabetes: A systematic review. J Health Psychol. 2018;1749-65. https://doi. org/10.1177/1359105316669580.

4. Streisand R, Mackey ER, Elliot BM, et al. Parental anxiety and depression associated with caring for a child newly diagnosed with type 1 diabetes: Opportunities for education and counseling. Patient Educ Couns. 2008;73(2):333-8. https://doi. org/10.1016/j.pec.2008.06.014.

5. Drotar D. Relating parent and family functioning to the psychological adjustment of children with chronic health conditions: what have we learned? What do we need to know? J Pediatr Psychol. 1997;22(2):149-65. https://doi. org/10.1093/jpepsy/22.2.149.

6. George C, Solomon J. The caregiving system: A behavioral systems approach to parenting. Handbook of attachment: Theory, research, and clinical applications 2008;2:833-56.

7. Bowlby J. Attachment and loss v. 3 (Vol. 1). Random House. Furman, W., \& Buhrmester, D. (2009). Methods and measures: The network of relationships inventory: Behavioral systems version. International Journal of Behavioral Development 1969;33:470-8.

8. Ainsworth MD, Blehar MC, Waters E,
Wall S. Patterns of attachment: Assessed in the strange situation and at home. 1978.

9. Ainsworth MD. Attachments across the life span. Bulletin of the New York Academy of medicine 1985;61(9):792.

10. Shaver PR, Mikulincer M. Attachmentrelated psychodynamics. Attach Hum Dev. 2002;4:133-61.

11. Main M, Hesse E. Attachment narratives and attachment across the lifespan. In Fall Meeting of the American Psychoanalytic Association, New York (Vol. 20). 1997.

12. Martínez C, Santelices MP. Evaluación del apego en el adulto: una revisión. Psykhe (Santiago) 2005;14(1):181-91.

13. Ciechanowski PS, Katon WJ, Russo JE, Walker EA. The patient-provider relationship: attachment theory and adherence to treatment in diabetes. Am J Psychiatry. 2001;158(1):29-35.

14. Moreira H, Canavarro MC. Parental attachment insecurity and parenting stress: The mediating role of parents' perceived impact of children's diabetes on 
the family. Families, Systems, \& Health 2016;34(3):240.

15. Gunnar M, Quevedo K. The neurobiology of stress and development. Annu Rev Psychol. 2007;58:145-73.

16. Fonagy P, Luyten P. A developmental, mentalization-based approach to the understanding and treatment of borderline personality disorder. Dev Psychopathol. 2009;21(4):1355-81.

17. McEwen BS. Physiology and neurobiology of stress and adaptation: central role of the brain. Physiol Rev. 2007;87(3):873904.

18. Sapolsky RM. The Physiological Relevance of Glucocorticoid Endangerment of the Hippocampus a. Ann N Y Acad Sci. 1994;746(1):294-304.

19. Greening L, Stoppelbein L, Konishi C, Jordan SS, Moll G. Child routines and youths' adherence to treatment for type 1 diabetes. J Pediatr Psychol. 2007;32(4):437-47. https://doi. org/10.1093/jpepsy/jsl029

20. Kelo M, Martikainen M, Eriksson E. Self-care of school-age children with diabetes: an integrative review. J Adv Nurs. 2011;67(10):2096-108. https://doi. org/10.1111/j.1365-2648.2011.05682.x

21. Gowers SG, Jones JC, Kiana S, North $\mathrm{CD}$, Price DA. Family functioning: a correlate of diabetic control? J Child Psychol Psychiatry. 1995;36(6):993-1001. Retrieved from https://www.ncbi.nlm.nih. gov/pubmed/7593406

22. Bearman KJ, La Greca AM. Assessing friend support of adolescents' diabetes care: the diabetes social support questionnaire-friends version. J Pediatr Psychol. 2002;27(5):417-28. https://doi. org/10.1093/jpepsy/27.5.417.

23. George C, Solomon J. The caregiving system: A behavioral systems approach to parenting. Handbook of attachment:
Theory, research, and clinical applications 2008;2:833-56.

24. Montecino S. Presencia y ausencia. Género y mestizaje en Chile. Editorial Catalonia. 1993.

25. Vertinsky P.Aging Bodies, Aging Sport Historians, and the Choreographing of Sport History. Sport History Rev. 1998;29(1):18-29. doi:10.1123/shr.29.1.18

26. Olmsted MP, Colton PA, Daneman D, Rydall AC, Rodin GM. Prediction of the onset of disturbed eating behavior in adolescent girls with type 1 diabetes. Diabetes Care. 2008;31(10):1978-82.

27. Nielsen S. Eating disorders in females with type 1 diabetes: an update of a meta-analysis. European Eating Disorders Review: The Professional Journal of the Eating Disorders Association 2002;10(4):241-54.

28. Hernández M, Benítez R, Medranda I, Pizarro C, Méndez MJ. Variaciones fisiológicas normales del desarrollo puberal: edad del inicio puberal, edad de la menarquia y talla. Anales de Pediatría 2008;69(2):147-53. https://doi. org/10.1157/13124894.

29. Durruty, Sansana. Hemoglobina glicosilada A1c como criterio diagnóstico de diabetes y pre-diabetes. Rev. Chil. Endocrinol. Diabetes 2011;4(1):38-43.

30. DiMeglio LA, Acerini CL, Codner E, et al. Clinical Practice Consensus Guideline 2018: Glycemic control targets and glucose monitoring for children, adolescents, and young adults with diabetes. Pediatr Diabetes. 2018 Oct;19 Suppl 27:105-114. doi: 10.1111/ pedi. 12737

31. Varni JW, Burwinkle TM, Jacobs JR, Gottschalk M, Kaufman F, Jones KL. The PedsQL ${ }^{\mathrm{TM}}$ in type 1 and type 2 diabetes: reliability and validity of the Pediatric Quality of Life Inventory ${ }^{\mathrm{TM}}$ generic core scales and type 1 diabetes module. Diabetes Care. 2003;26(3):631-7.

32. Delamater AM, de Wit M, McDarby V, et al. ISPAD Clinical Practice Consensus Guidelines 2018: Psychological care of children and adolescents with type 1 diabetes. Pediatr Diabetes. 2018;19 Suppl 27:237-49. https://doi.org/10.1111/ pedi.12736

33. Brennan KA, Clark CL, Shaver PR. Selfreport measurement of adult attachment: An integrative overview. 1998.

34. Spencer R, Guzmán M, Fresno A, Ramos $\mathrm{N}$. Validación chilena del cuestionario de evaluación del apego romántico Experiences in Close Relationships (ECR): análisis de la validez de criterio. Terapia psicológica 2013;31(3):313-24.

35. Cohen S, Kamarck T, Mermelstein R. A global measure of perceived stress. J Health Soc Behav. 1983;385-96.

36. Calderón-Carvajal C, Gómez N, López F, Otárola N, Briceño M. Estructura factorial de la escala de estrés percibido (PSS) en una muestra de trabajadores chilenos. Salud \& Sociedad 2017;8(3):218-26.

37. Kerns KA, Klepac L, Cole A. Peer relationships and preadolescents' perceptions of security in the childmother relationship. Developmental psychology 1996;32(3):457.

38. Cárdenas-Fernandez. (s.f). Psychometric adaptation of the security scale in middle childhood (9-11 years old) in a state school in Los Olivos.

39. Osika W, Friberg P, Wahrborg P. A new short self-rating questionnaire to assess stress in children. Int J Behav Med. 2007;14(2):108-17.

40. Caqueo-Urízar A, Urzúa A, Osika W. Psychometric properties of the Spanish language version of the stress in children questionnaire (SiC). Psicologia: Reflexão e Crítica 2014;27(1):81-9. 